\title{
Initial colonization, community assembly and ecosystem function: fungal colonist traits and litter biochemistry mediate decay rate
}

\author{
LAUREN C. CLINE* and DONALD R. ZAK*† \\ *School of Natural Resources E Environment, University of Michigan, 440 Church St., Ann Arbor, MI 48109, USA, \\ $\dagger$ Department of Ecology E Evolutionary Biology, University of Michigan, 440 Church St., Ann Arbor, MI 48109, USA
}

\begin{abstract}
Priority effects are an important ecological force shaping biotic communities and ecosystem processes, in which the establishment of early colonists alters the colonization success of later-arriving organisms via competitive exclusion and habitat modification. However, we do not understand which biotic and abiotic conditions lead to strong priority effects and lasting historical contingencies. Using saprotrophic fungi in a model leaf decomposition system, we investigated whether compositional and functional consequences of initial colonization were dependent on initial colonizer traits, resource availability or a combination thereof. To test these ideas, we factorially manipulated leaf litter biochemistry and initial fungal colonist identity, quantifying subsequent community composition, using neutral genetic markers, and community functional characteristics, including enzyme potential and leaf decay rates. During the first 3 months, initial colonist respiration rate and physiological capacity to degrade plant detritus were significant determinants of fungal community composition and leaf decay, indicating that rapid growth and lignolytic potential of early colonists contributed to altered trajectories of community assembly. Further, initial colonization on oak leaves generated increasingly divergent trajectories of fungal community composition and enzyme potential, indicating stronger initial colonizer effects on energy-poor substrates. Together, these observations provide evidence that initial colonization effects, and subsequent consequences on litter decay, are dependent upon substrate biochemistry and physiological traits within a regional species pool. Because microbial decay of plant detritus is important to global $\mathrm{C}$ storage, our results demonstrate that understanding the mechanisms by which initial conditions alter priority effects during community assembly may be key to understanding the drivers of ecosystem-level processes.
\end{abstract}

Keywords: assembly history, community assembly, decomposition, fungi, priority effects

Received 25 June 2015; revision received 17 August 2015; accepted 24 August 2015

\section{Introduction}

Community assembly history, or the stochastic sequence and timing of species arrival, is an important ecological force shaping competitive outcomes, and, in turn, the composition of biotic communities (Lewontin 1969; Diamond 1975; Drake 1991; Chase 2003). Initial colonizers can exclude later-arriving species, a mechanism

Correspondence: Lauren C. Cline, Fax: 734-936-2195;

E-mail: clinela@umich.edu known as priority effects, as a result of strong interspecific interactions and habitat modification (Wilbur \& Alford 1985; Belyea \& Lancaster 1999; Vannette \& Fukami 2014). For example, gaining early access to resources can lead to niche pre-emption by an initial colonist and the subsequent competitive exclusion of later-arriving species (Körner et al. 2008). Furthermore, through resource consumption and the production of secondary metabolites, initial colonists can suppress the colonization success of later-arriving species (Allison 2012; Hiscox et al. 2015). Due to the competitive 
advantage obtained by initial colonizers, early immigration history appears to have important consequences for ecosystem-level processes, including biogeochemical cycling in soils (Körner et al. 2008; Fukami et al. 2010; Dickie et al. 2012). Furthermore, priority effects could be particularly important in litter-decay systems as autumnal leaf abscission results in large litter inputs atop an established saprotrophic community.

Despite our growing knowledge of the mechanisms by which historical contingencies shape community assembly, the magnitude of priority effects appears dependent on individual species and environmental conditions (Chase 2007; Kardol et al. 2013; Tucker \& Fukami 2014; Hiscox et al. 2015). For example, strong priority effects occurred in a field manipulation of wood-decay fungi, yet the introduction of some species elicited larger changes to community composition and rates of wood decomposition relative to others (Dickie et al. 2012). However, we do not understand which traits of initial colonists or environmental conditions lead to strong priority effects, and there have been few empirical tests of their effect on community assembly and ecosystem processes (Vannette \& Fukami 2014).

The strength of priority effects may depend on the physiological traits of initial colonists. For example, rapidly growing organisms may gain a larger competitive foothold when they are the initial colonists of a habitat (Vannette \& Fukami 2014; Cleland et al. 2015). Additionally, initial colonists capable of accessing energy-poor resources, such as lignin during leaf decay, could gain a substantial advantage in the absence of rapidly growing competitors. The physiological capacity to decompose lignin, an aromatic biopolymer and major constituent of plant litter, is largely limited to a small subset of micro-organisms in the fungal phylum Basidiomycota (Hatakka 1994; Floudas et al. 2012). By gaining access to a resource largely inaccessible to other decay organisms, initial colonization by lignolytic fungi could plausibly alter the trajectory of community assembly. Furthermore, an organism's ability to produce secondary metabolites may enhance priority effects through the combination of interference competition and habitat modification (Boddy 2000; Kennedy \& Bruns 2005; Hiscox et al. 2015). Strong priority effects may also occur between more closely related taxa, due to the intensity of competition between ecologically similar species, as observed in model yeast and bacterial communities (Peay et al. 2012; Tan et al. 2012). By considering a colonist's resource requirements, potential to modify the environment, and resource use overlap with other species, Vannette \& Fukami (2014) demonstrate the utility of incorporating species traits to model priority effects in nectar-inhabiting yeast communities with varied assembly history.
In addition to physiological traits of initial colonists, local resource availability may serve to magnify or dampen the strength of priority effects by directly impacting the colonization success of early-arriving propagules (Chase 2007; Langenheder \& Székely 2011; Pagaling et al. 2014). For example, conditions of low resource supply may decrease the successful establishment of initial colonists, thereby overriding priority effects with strong selection imposed by the local environment (habitat filtering sensu Chase 2007). Whereas, under high resource availability, priority effects may be enhanced as initial colonizers establish quickly and exclude later-arriving species (Ejrnæs et al. 2006; Kardol et al. 2013). Identifying species traits and habitat characteristics that lead to strong priority effects, via the successful colonization of early colonists, may be key to understanding drivers of community assembly, especially diverse communities of saprotrophic microorganisms in soil (Nemergut et al. 2013; Van der Wal et al. 2013).

Moreover, understanding the combined importance of early immigration history and habitat filtering during community assembly may elucidate the complex linkages between composition and function. Early immigration history may shape rates of ecosystem processes, if compositional differences elicited by initial colonization reflect important functional trait differences in the community. For example, the order of colonization by wood-decay fungi altered rates of decomposition in both laboratory and field settings (Fukami et al. 2010; Dickie et al. 2012), indicating direct links between early immigration history, community composition and functional characteristics of the community. Alternatively, if habitat filtering remains a more important ecological force shaping the functional traits of communities, functional convergence may occur during community assembly despite divergence in composition (Fukami et al. 2005).

Using saprotrophic litter-decay fungi as a model system, we implemented a microcosm experiment to investigate how physiological traits of initial colonizers interact with litter biochemistry to influence early immigration history. Because saprotrophic micro-organisms have varied capacities to degrade constituents of plant detritus, litter biochemistry functions as a strong habitat filter (McGuire et al. 2010; Voříšková \& Baldrian 2013). We evaluated initial colonization effects by quantifying the variation in composition and function of fungal litter-decay communities previously colonized, relative to 'control' communities receiving no initial colonizer. Due to ecological similarities, we expected that the initial colonization of closely related fungi would result in the assembly of similar communities through time. Further, we hypothesized that initial colonizers exhibiting rapid 
growth and/or high lignolytic capacity would result in larger deviations in community composition and function, relative to a control community. Additionally, we reasoned that resource availability alters the effects of initial colonization, such that the impact of a particular initial colonizer on community assembly would vary on lignin-poor and lignin-rich leaf litter. We further hypothesized that the importance of an initial colonizer would attenuate with time due to the increased importance of habitat filtering as the biochemical components of plant litter are metabolized and lignin dominates the latter stages of decay. To test these ideas, we factorially manipulated combinations of leaf litter biochemistry and initial fungal colonization to quantify community assembly and leaf decay throughout an 8-month laboratory experiment, the equivalent of a growing season in temperate forests. Furthermore, we carefully characterized initial colonizers to investigate the physiological traits resulting in varied fungal community composition and rates of decomposition.

\section{Materials and methods}

\section{Experimental design $\mathcal{E}$ sampling}

To understand the relative importance of the physiological traits of initial fungal colonists and habitat filtering in shaping community assembly, we collected leaves of contrasting biochemistry and cultured litter-decay fungi from a northern hardwood (NH) ecosystem near Oceana, MI. This ecosystem was chosen because it is a widespread forest type of North America. Further, previous characterizations of litter-decay fungi at this site provided the background knowledge to experimentally manipulate native, ecologically relevant fungal communities (Edwards \& Zak 2011; Entwistle et al. 2013; Cline \& Zak 2014). Leaf litter traps were placed in the field to collect senescent leaves of Acer saccharum and Quercus rubra (hereafter, maple and oak litter). Biochemical analyses revealed that oak leaf litter had over twice the lignin content of maple leaves, as well as a higher C:N (Table S1 in Supporting information). Leaf lignin content was determined by the acid detergent lignin (ADL) procedure, in which ADL was determined gravimetrically as the residue remaining upon ignition after $\mathrm{H}_{2} \mathrm{SO}_{4}$ treatment (Goering \& Van Soest 1970). Leaf cellulose was calculated by subtracting percentage acid detergent fibre (ADF) and lignin from dry mass. ADF was determined gravimetrically as the residue remaining after dissolution and extraction of cell solubles, hemicellulose and soluble minerals with hexadecyltrimethylammonium bromide and sulphuric acid. Total leaf C was determined using a CNS2000 Analyzer $\left(\right.$ LECO $^{\circledR}$ St. Joseph, MI, USA). Total leaf $\mathrm{N}$ was mea- sured colorimetrically following digestion in concentrated $\mathrm{H}_{2} \mathrm{SO}_{4}$ (Lachet Instruments, Loveland, CO, USA).

We generated an isolate collection of prevalent litterdecay fungi, following the collection of sporocarps and decaying leaves from our study site in September 2013. Furthermore, prevalent ascomycete saprotrophs present in prior molecular inventories of this study site (Edwards \& Zak 2011; Entwistle et al. 2013; Cline \& Zak 2014), but missing from the culture collection, were obtained from the USDA Forests Products Laboratory. From a collection of 30 fungal isolates, 6 initial colonists were carefully chosen to represent phylogenetic pairs of fungi with varied metabolic capacities (Fig. 1), including Aspergillus asperescens, Dichostereum aff. Pallescens, Gymnopus contrarius, Mycena galopus, Rhodocollybia butyraceae and Phomopsis sp. Phylogenetic pairs of fungi were determined by constructing a maximum-likelihood phylogenetic tree following DNA extraction and amplification of the fungal 28S gene (protocols located in the following section). For a list of reference sequences used to construct the phylogenetic tree, see Table S2 (Supporting information). Species-level designations of isolates were made according to top blast hits with $>99 \%$ maximum identity and an e-value of 0 . To measure colonist respiration rates, each organism was inoculated onto oak and maple leaf treatments, and respiration was quantified for 2 weeks using a gas chromatograph equipped with a Porapak $Q$ column and a thermal conductivity detector (Trace 2000, Thermo Quest, CA, USA). On both litter types, respiration was highest for Phomopsis and lowest in Rhodocollybia (Fig. 1B). Here, and in following sections, we refer to fungal isolates by genus for simplicity. However, fungal isolates represent a single genetic individual; therefore, isolate characteristics and initial colonization effects are not necessarily generalizable to species or genus effects.

To compare the potential enzyme activity of fungal colonists on sterile oak and maple litter, $0.5 \mathrm{~g}$ of homogenized leaves were sampled and assayed for the activity of $\beta$-1,4-glucosidase, cellobiohydrolase, $\mathrm{N}$-acetyl- $\beta$-glucosaminidase, and summed phenol oxidase and peroxidase activity. To measure activity of $\beta-1$, 4-glucosidase, cellobiohydrolase and $\mathrm{N}$-acetyl- $\beta$-glucosaminidase, we used $200 \mu \mathrm{M}$ methylumbelliferyl MUB-linked substrates. A 25-mM L-dihydroxy-phenylalanine (L-DOPA) substrate was used to assay phenol oxidase and peroxidase potential. Enzyme activity was measured in a Molecular Devices $\mathrm{f}$ MAX fluorometer set at $365 \mathrm{~nm}$ excitation wavelength and $460 \mathrm{~nm}$ emission wavelength. Phenol oxidase and peroxidase assays were incubated for $24 \mathrm{~h}$, and rates were estimated spectrophotometrically (Saiya-Cork et al. 2002). Euclidean distances of log-transformed enzyme activity were calculated to visualize variation in colonizer enzymatic 
A
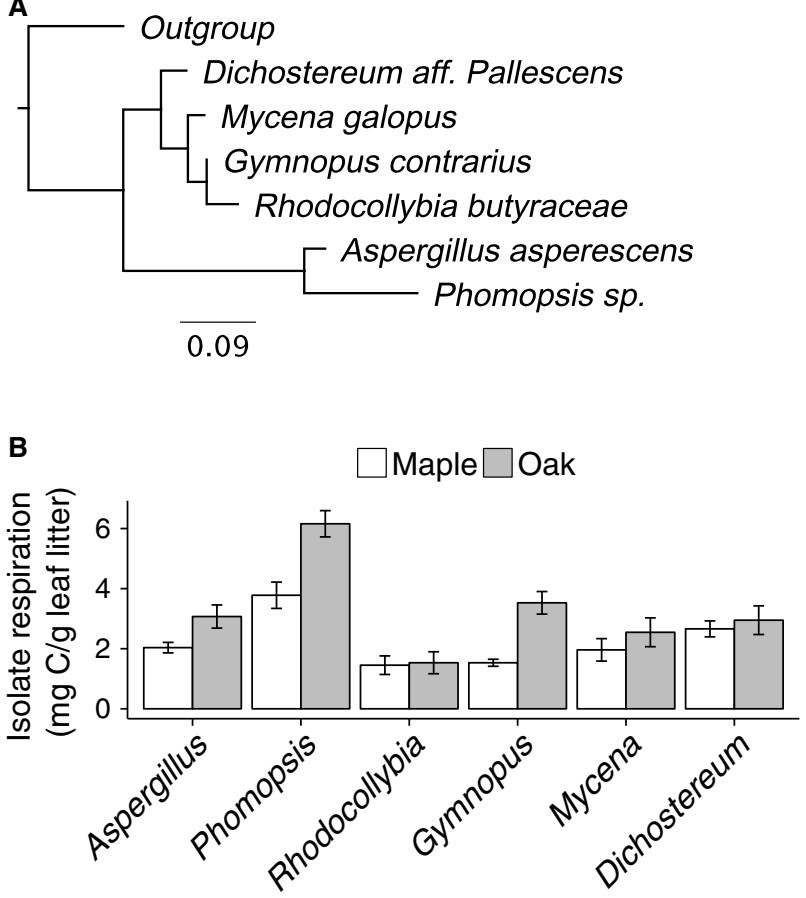

C

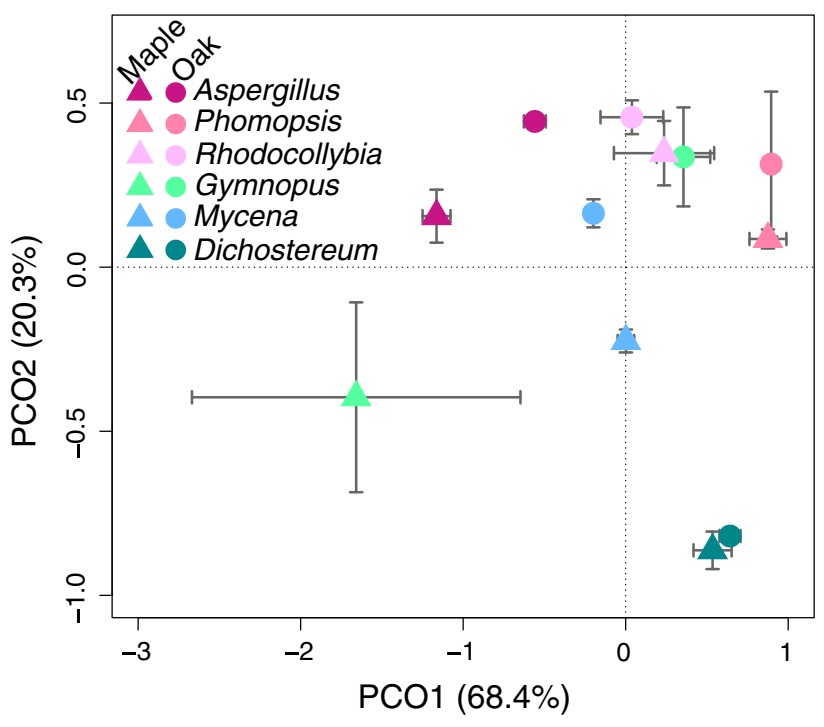

Fig. 1 Six initial colonizers were selected according to varied evolutionary histories (A), rates of respiration (B) and potential enzyme activity (C). A maximum-likelihood phylogenetic tree was constructed following the amplification of a fragment of the $28 \mathrm{~S}$ fungal gene from each colonizer. Respiration of initial colonizers growing on sterile leaf litter was quantified using a gas chromatogram $(n=15)$, for a period of two weeks, prior to the inoculation of the native litter community. Open bars represent maple leaf litter treatments and closed bars oak treatments. Error bars denote standard error. Potential activity of each enzyme category was logtransformed, followed by the calculation of pairwise Euclidean distance between samples, and visualization by principal coordinates analysis (PCoA). Similarly in the PCoA, error bars represent standard error between replicates within a treatment.

potential (principal coordinates analysis; Fig. 1C). PCo1 correlated with $\beta$-glucosidase, $\mathrm{N}$-acetylaminoglucosidase and cellobiohydrolase activity $(r=0.89-0.91$, $P<0.0001)$ and PCo2 correlated with lignolytic activity ( $r=-0.88, P<0.0001)$, illustrating the high lignolytic potential of Dichostereum, as well as the potential of Phomopsis to metabolize cellulose and chitin.

To investigate the consequences of niche pre-emption by an initial colonist and habitat filtering, experimental microcosms were constructed using two contrasting litter types, which were subsequently inoculated with the initial colonists described above. Microcosms consisted of $250-\mathrm{mL}$ wide-mouth jars containing $2.5 \mathrm{~g}$ of maple or oak leaves atop $70 \mathrm{~g}$ of acid-washed, autoclaved sand (Quikrete, MI, USA). Leaves were dried at $40{ }^{\circ} \mathrm{C}$, cut into $1 \mathrm{~cm}^{2}$ squares and sterilized by ethylene oxide fumigation (STERIS, MN, USA). Prior to experimentation, sand was saturated and dry litter was wetted with deionized, sterile water. We manipulated initial colonizer history by inoculating a single fungal colonist onto sterile leaves, allowing colonist establishment (14 days), and then introducing a native saprotrophic community. Fourteen days was chosen as a slightly shortened establishment period relative to experimental tests of priority effects in wood-decay fungal communities (Fukami et al. 2010; Dickie et al. 2012). To determine consequences of niche pre-emption, a control treatment received no initial colonizer prior to introduction of the native community. Initial colonizers were introduced to each microcosm using two agar plugs from fungal cultures. The native community was extracted from decaying litter collected from our field site. Briefly, $50 \mathrm{~g}$ of leaf litter and $500 \mathrm{~mL}$ of autoclaved deionized water was homogenized in a blender for $1 \mathrm{~min}$ and filtered through a $500-\mu \mathrm{m}$ filter to obtain a homogenous suspension (He et al. 2010). One millilitre of this slurry was added to each microcosm. Microcosms were maintained at $20{ }^{\circ} \mathrm{C}$ and $65 \%$ water-holding capacity, within the favourable range for saprotrophic activity (Langenheder \& Prosser 2008). A total of 210 microcosms provided five replicates for two litter types and seven initial colonizer histories (including the control), which we harvested at 3 time points. Microcosms were destructively harvested at 1, 3 and 8 months following addition of the native community. At each harvest, leaf mass was determined, then homogenized using sterile scissors. A 
$0.5 \mathrm{~g}$ sample was removed and placed at $4{ }^{\circ} \mathrm{C}$ for enzyme assays, whereas the remaining sample was stored at $-80{ }^{\circ} \mathrm{C}$ for molecular community analysis.

\section{DNA extraction $\mathcal{E}$ community analysis}

Targeted amplification of the fungal large ribosomal subunit (28S) was performed to characterize community composition. After each harvest, total DNA was isolated from two replicates of each microcosm using the MoBio PowerLyzer DNA Extraction kit. DNA was extracted from $0.25 \mathrm{~g}$ of leaf litter and stored at $-80{ }^{\circ} \mathrm{C}$, until we could initiate PCR amplification. Fungal richness and $\beta$ diversity were estimated by targeting the $28 \mathrm{~S}$ gene using primers LROR and LR3 (Vilgalys \& Hester 1990). Primers were selected to capture the D1 and D2 hypervariable regions of the $28 \mathrm{~S}$ gene, increasing the accuracy of taxonomic assignment while also allowing for phylogenetic analyses (Liu et al. 2012; Porter \& Golding 2012). Triplicate PCRs for each sample contained: $400 \mu \mathrm{M}$ primers, $200 \mu \mathrm{M}$ dNTPs, $1.5 \mathrm{mM} \mathrm{MgCl}_{2}$, $0.01 \mathrm{mg}$ BSA and 2U Taq polymerase. Following an initial denaturation step at $95^{\circ} \mathrm{C}$ for $5 \mathrm{~min}$, PCR was cycled 30 times at $95{ }^{\circ} \mathrm{C}$ for $30 \mathrm{~s}, 54{ }^{\circ} \mathrm{C}$ annealing temperature for $30 \mathrm{~s}, 72{ }^{\circ} \mathrm{C}$ for $75 \mathrm{~s}$ and a final extension at $72{ }^{\circ} \mathrm{C}$ for $7 \mathrm{~min}$. PCR products were purified using Qiagen MinElute PCR kit and quantified using PicoGreen dsDNA kit. Sequencing was performed on the PacBio RS II system utilizing circular consensus technology, which can generate 99.5-99.9\% sequence accuracy for DNA fragments ranging from 150 to $500 \mathrm{bp}$ (Travers et al. 2010). To enable multiplexing of samples, a 16nucleotide barcode was added to the $5^{\prime}$ end of each forward and reverse primer. Ten barcoded samples, pooled in equimolar concentrations, were multiplexed on each SMRT chip. Twenty-one total SMRT chips were analysed at the University of Michigan Sequencing Facility. Sequences were processed in Mothur using established pipeline procedures (Schloss et al. 2011), aligned to $28 \mathrm{~S}$ reference alignments (Cole et al. 2014), and chimeras were identified using uchime (Edgar et al. 2011). Operational taxonomic units (OTUs) were clustered at $99 \%$ sequence similarity (Martiny et al. 2011), and taxonomic identity was determined using the RDP classifier. Each sample was rarefied to 500 sequences; 10 samples failed to meet the sequence count and were excluded from analysis. Due to recent concerns regarding the statistical validity of subsampling in conjunction with next generation sequencing platforms (McMurdie \& Holmes 2014), we also analysed sequence data by normalizing OTU abundance to proportions of total sequences. Because we obtained similar results (Table S3, Supporting information), rarefied sequence data are presented. Observed OTU richness was used to compare $\alpha$-diversity between samples. Good's coverage was employed as an estimator of sampling completeness, calculating the probability that a randomly selected amplicon had already been sequenced (Good 1953; Claesson et al. 2009).

In total, 15181 unique sequences were obtained, ranging in length from 493 to $632 \mathrm{bp}$. Sequences were assigned to phyla Ascomycota (73.6\%), Basidiomycota $(19.8 \%)$, Fungi incertae sedis $(6.5 \%)$ and a small number of Chytridiomycota $(0.1 \%)$. The most abundant ascomycete orders consisted of Hypocreales (26\%) and Eurotiales (24\%), whereas Agaricales (15\%) and Polyporales (3\%) comprised the most abundant basidiomycete orders. Observed OTU richness ranged from 23 to 145 OTUs per sample. Good's coverage estimates ranged from 0.71 to 0.98 , indicating that communities were undersampled, although the most abundant members of the fungal community were captured. Following logtransformation of OTU relative abundance, taxonomic $\beta$-diversity was calculated using the Bray-Curtis dissimilarity metric. Sequences were uploaded to the NCBI Sequence Read Archive under study Accession no. SRP056628.

\section{Functional analysis}

To characterize litter decomposition, we quantified remaining leaf mass, microbial respiration and potential enzyme activity. Leaf mass was calculated as ash-free mass remaining after 1, 3 and 8 months of decomposition. Following the addition of the native saprotrophic community to each microcosm, respiration was quantified weekly (according to protocol described above) for the first 3 months of the experiment. After headspace gas was sampled, the lids of the microcosms were removed for $30 \mathrm{~min}$ under a sterile hood to equalize $\mathrm{CO}_{2}$ with the ambient atmosphere. Using the $\mathrm{R}$ package grofit, cumulative respiration was fit to the Sigmoidal Gompertz model (Zwietering et al. 1990) to estimate the length of lag time $(\lambda)$, maximum respiration rate $(\mu)$ and amount of substrate available for metabolism $(A)$. Protocols to quantify enzyme potential of litter communities, at 1, 3 and 8 months, described above, were conducted immediately following destructive harvesting. Following log-transformation of potential activity of each enzyme category, pairwise Euclidean distances were calculated for multivariate analysis.

\section{Statistical analysis}

Univariate and multivariate statistics were employed to quantify the importance of initial colonizer history, habitat filtering and colonizer traits in shaping the assembly of saprotrophic microbial communities. To 
identify whether initial colonizer history resulted in parallel changes in fungal communities and decomposition dynamics, Mantel tests quantified matrix correlations between fungal $\beta$-diversity (Bray-Curtis distance) and Euclidean dissimilarities in mass loss and enzyme potential between treatments. Analysis of variance (ANOVA) determined whether initial colonizer history and litter biochemistry influenced OTU richness, respiration and mass loss at each time point. For multivariate variables, fungal $\beta$-diversity and Euclidean differences in community enzymatic potential, we conducted permutational multivariate analysis of variance (PERMANOVA) following 9999 permutations. To determine whether the impact of initial colonizer history varied across contrasting litter types, we identified significant interactions between factors in both ANOVA and PERMANOVA models. To test the hypothesis that the importance of initial colonization history attenuated through time, effect sizes of factors were calculated as partial eta-squared $\left(\eta_{p}^{2}\right)$, or the variation explained by a factor in relation to the summed variation explained by the factor and the error associated with the model (Lakens 2013). Due to the limitations of $R^{2}$ in comparing effect sizes between models (Nakagawa \& Cuthill 2007), this metric was selected to compare effect sizes within in model, as well as across time points. Partial eta-squared is calculated as follows:

$$
\eta_{p^{2}}=\frac{S S_{\text {factor }}}{S S_{\text {factor }}+S S_{\text {resid }}}
$$

We employed dispersion analysis for $\beta$-diversity and Euclidean variation in enzyme potential to quantify the variability elicited by initial colonizer history between litter types. Specifically, dispersion was calculated by the average dissimilarity of communities with different fungal colonists to the centroid of all oak and maple litter communities. To evaluate whether outcomes would be similar for closely related colonists, Mantel tests quantified correlations between colonist phylogenetic distance and fungal $\beta$-diversity. To test the hypothesis that colonist respiration rate and lignolytic potential shaped assembling communities, linear regression was conducted between colonist characteristics and BrayCurtis dissimilarities, Euclidean distances and mass loss relative to control communities. These departures from controls were calculated for each litter type, resulting in 12 comparisons. To aid in community analysis, similarity percentage analysis (SIMPER) calculated the contribution of OTUs towards the community dissimilarity between each initial colonizer and control communities. Assumptions of linearity were verified prior to conducting linear regression and ANOVA, followed by necessary transformations. Statistical tests were conducted using the $\mathrm{R}$ packages vegan (Oksanen et al. 2015) and grofit (Kahm et al. 2010; http:/ / www.R-project.org).

\section{Results}

Initial colonization history shapes community assembly

With respect to the fungal communities developing on oak and maple leaves (hereafter 'oak litter communities' and 'maple litter communities'), initial fungal colonizers significantly influenced $\beta$-diversity, litter decay and enzyme potential at each time point (Table 1). Averaged across litter type, the highest OTU richness occurred in control communities receiving no initial colonizer, followed by litter communities initially colonized with Rhodocollybia, Aspergillus, Phomopsis, Dichostereum, Gymnopus and finally Mycena (Table S4, Supporting information). Tukey's HSD revealed that after one month, oak litter communities initially colonized by Gymnopus had a significantly lower OTU richness (38 \pm 9 OTUs) relative to control oak litter community (116 \pm 12 OTUs; $P<0.00002)$, whereas maple litter communities initially colonized by Mycena had a significantly lower OTU richness (43 \pm 9 OTUs) relative to control maple litter community (110 \pm 7 OTUs; $P=0.005)$. After 3 months, oak litter communities initially colonized by Mycena had a significantly lower

Table 1 Effect sizes $\left(\eta_{\mathrm{p}}{ }^{2}\right)$ from ANOVA (OTU richness, mass loss) and PERMANOva ( $\beta$-diversity and enzyme potential) models at each experimental time point. $\eta_{p}^{2}$ was calculated as the proportion of variation explained by factor when accounting for error in model. Fungal $\beta$-diversity was calculated from the Bray-Curtis dissimilarity metric. Variation in enzyme potential was calculated as pairwise Euclidean distances of 5 extracellular enzymes (see Methods for more details)

\begin{tabular}{lllll}
\hline $\begin{array}{l}\text { Response } \\
\text { variable }\end{array}$ & Factor & 1 month & 3 months & 8 months \\
\hline OTU richness & Colonizer & $0.45^{\dagger}$ & $0.60^{\dagger}$ & $0.51^{\ddagger}$ \\
& Litter type & 0.01 & 0.02 & 0.001 \\
& $\mathrm{C} \times \mathrm{L}$ & $0.41^{\dagger}$ & $0.19^{\ddagger}$ & $0.35^{\ddagger}$ \\
$\beta$-diversity & Colonizer & $0.31^{\dagger}$ & $0.26^{\dagger}$ & $0.26^{\dagger}$ \\
& Litter type & $0.06^{\dagger}$ & $0.08^{\dagger}$ & $0.12^{\dagger}$ \\
& $\mathrm{C} \times \mathrm{L}$ & $0.15^{\dagger}$ & $0.12^{\dagger}$ & $0.16^{\dagger}$ \\
Enzyme & Colonizer & $0.58^{\dagger}$ & $0.42^{\dagger}$ & $0.49^{\dagger}$ \\
potential & Litter type & $0.45^{\dagger}$ & $0.46^{\dagger}$ & $0.68^{\dagger}$ \\
& $\mathrm{C} \times \mathrm{L}$ & $0.25^{\ddagger}$ & $0.20^{\S}$ & $0.32^{\ddagger}$ \\
Mass loss & Colonizer & $0.21^{\S}$ & $0.30^{\dagger}$ & $0.47^{\ddagger}$ \\
& Litter type & $0.54^{\dagger}$ & $0.30^{\dagger}$ & $0.17^{\ddagger}$ \\
& $\mathrm{C} \times \mathrm{L}$ & $0.19^{\dagger}$ & $0.20^{\S}$ & $0.30^{\ddagger}$ \\
\hline
\end{tabular}

$\mathrm{C} \times \mathrm{L}$ indicates the interaction term between colonizer and litter type.

†Represents factor significance at $\alpha<0.001,{ }^{\star} \alpha<0.01,{ }^{\S} \alpha<0.05$, and ${ }^{{ }^{\top}} \alpha<0.10$. 
average richness ( $23 \pm 1$ OTUs), relative to the control community growing on oak litter $(134 \pm 13$ OTUs; $P$ $<0.00001)$. After 8 months, significantly lower OTU richness was reported for oak litter communities initially colonized by Gymnopus (55 \pm 19 OTUs) and Mycena (34 \pm 9 OTUs) relative to control oak litter community (131 \pm 11 OTUs; $P<0.0016)$. Together, results indicate initial colonization by certain initial colonists can suppress fungal community richness through time.

Across both litter types, the wide range of community dissimilarity (Bray-Curtis distances) relative to control communities indicated that outcomes of initial colonization were dependent on colonist identity (Table S4, Supporting information). For example, the initial colonization of Mycena consistently resulted in large community dissimilarity relative to control communities growing on oak and maple litter, whereas colonization by Rhodocollybia did not alter community composition (Fig. 2). Not surprisingly, taxonomic assignment of OTUs contributing to differences in community composition between control and initial colonizer treatments (SIMPER) indicated that initial colonists were more abundant after one month, relative to control communities (Table S5, Supporting information). In addition, the presence of certain initial colonizers enhanced rates of oak and maple litter decomposition relative to their respective control community. For example, initial colonization by Phomopsis significantly decreased the lag phase of respiration (Table S6, Supporting information), indicating this colonist led to most rapid initial decay. Further, oak litter communities initially colonized by Gymnopus, Mycena and Dichostereum had higher maximum rates of respiration $(\mu)$, a greater substrate pool (A), and a greater rate of decay as revealed by litter mass loss (Fig. 3, Table S6, Supporting information). Similarly, maple litter communities inoculated with Mycena had significantly larger pools of metabolizable substrate and a lower remaining litter mass, relative to the control community growing on maple litter. Finally, lignolytic potential was enhanced in oak litter communities initially colonized with Gymnopus, Mycena and Dichostereum, as well as maple litter communities colonized with Mycena, as indicated by distinct separation of $\mathrm{PCo} 2$ in Fig. $4 \mathrm{C}$, an axis negatively correlated with lignolytic activity $(r=-0.99, P<0.0001)$. Together, results indicate that initial colonists, particularly basidiomycetes with high lignolytic potential, resulted in diverging community composition and enhanced rates of decay.

To understand whether initial colonizer history had a consistent effect on fungal community and functional characteristics, we conducted Mantel correlation tests between $\beta$-diversity, mass loss and enzyme potential of oak and maple litter communities. While no significant correlation occurred between variation in mass loss and $\beta$-diversity during the first and third months of the experiment $(P=0.25-0.56)$, distance matrices were significantly correlated following 8 months $\left(R_{\text {Mantel }}=0.26\right.$,
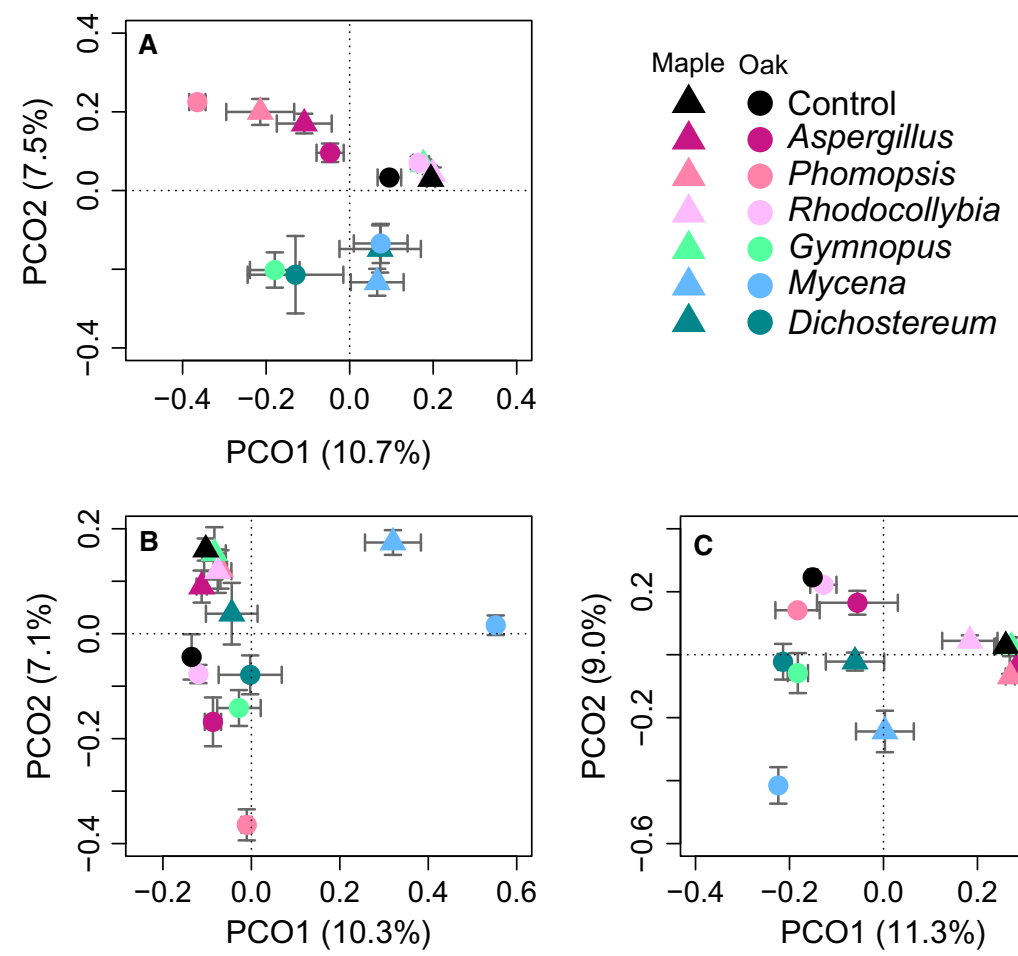

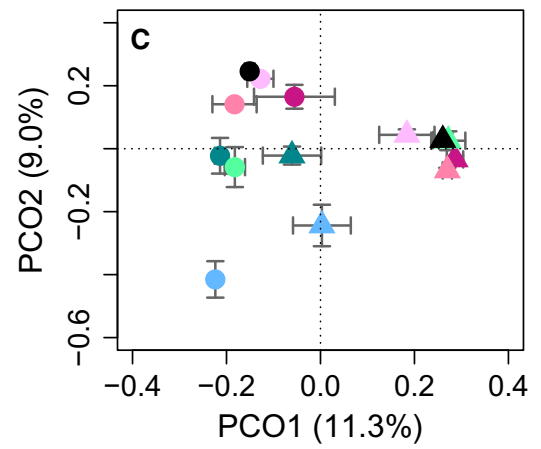

Fig. 2 Principal coordinates analysis of fungal $\beta$-diversity after 1 month (A), 3 months (B) and 8 months (C). The BrayCurtis distance metric was used to calculate pairwise differences in log-transformed OTU abundances between treatments. Error bars denote standard error between replicates within a treatment. 


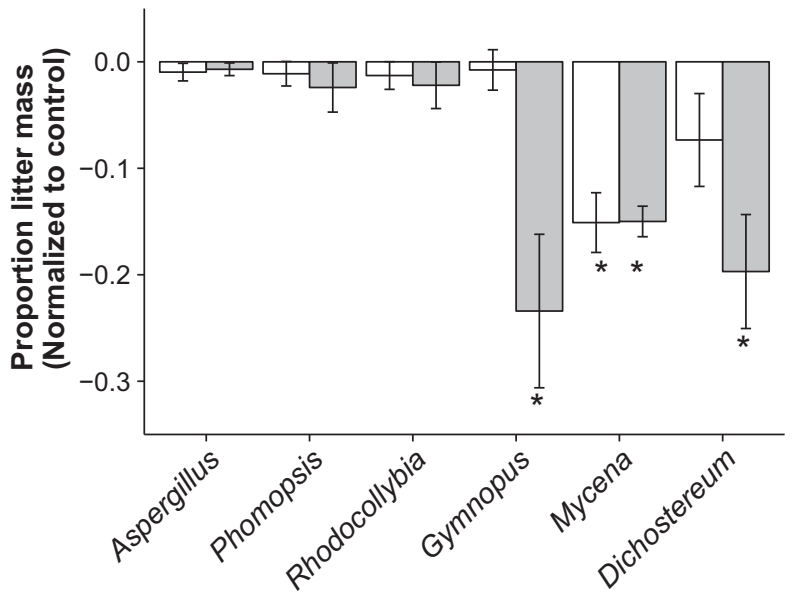

Fig. 3 Mass remaining after 8 months, normalized to the control community, on maple (open bars) and oak (closed bars) litter. Negative values indicate greater decay rates in litter communities inoculated with an initial colonist relative to the control. Error bars denote standard error. Representing values significantly different from zero, an asterisk denotes significance at $\alpha<0.05$.

$P=0.045)$. This result indicated that the initial colonist had parallel effects on community composition and metabolic rate during late stages of decay. Weak correlations occurred between $\beta$-diversity and variation in enzyme potential after 1 month $\left(R_{\text {Mantel }}=0.13\right.$, $P=0.098)$ and 8 months $\left(R_{\text {Mantel }}=0.23, \quad P=0.054\right)$, whereas no significant correlation occurred after 3 months $(P=0.23)$. Overall, evidence generally supported our hypothesis that changes to fungal communities, as a result of initial colonization, resulted in corresponding consequences to litter decay.

\section{Litter type alters consequences of initial colonizer history}

Consequences of initial colonization on fungal community composition and function were dependent on litter type, as indicated by the significant interaction terms for models of fungal $\beta$-diversity, litter decay and enzyme potential (Table 1). Dispersion analysis indicated that litter community composition was more variable on oak leaves relative to maple litter after 3 months (Fig. 2B, Pseudo- $\mathrm{F}_{1,68}=5.73, P=0.019$ ) and 8 months (Fig. 2C, Pseudo- $F_{1,58}=5.82, P=0.019$ ). Similarly, the initial fungal colonizer had a larger effect on the potential enzyme activity of oak litter communities, demonstrated by significantly greater dispersion in enzyme potential of oak litter communities relative to those growing on maple litter at each time point (Fig. 4, Pseudo- $F=4.91-13.8, P<0.001)$. Lastly, initial colonization by lignolytic fungi (i.e., Gymnopus, Mycena and Dichostereum) enhanced maximum rates of respiration $(\mu)$, substrate pool size (A) and decay rate (following 8 months) on oak litter, although only minor enhancements were observed on maple leaves (Table S6, Sup-
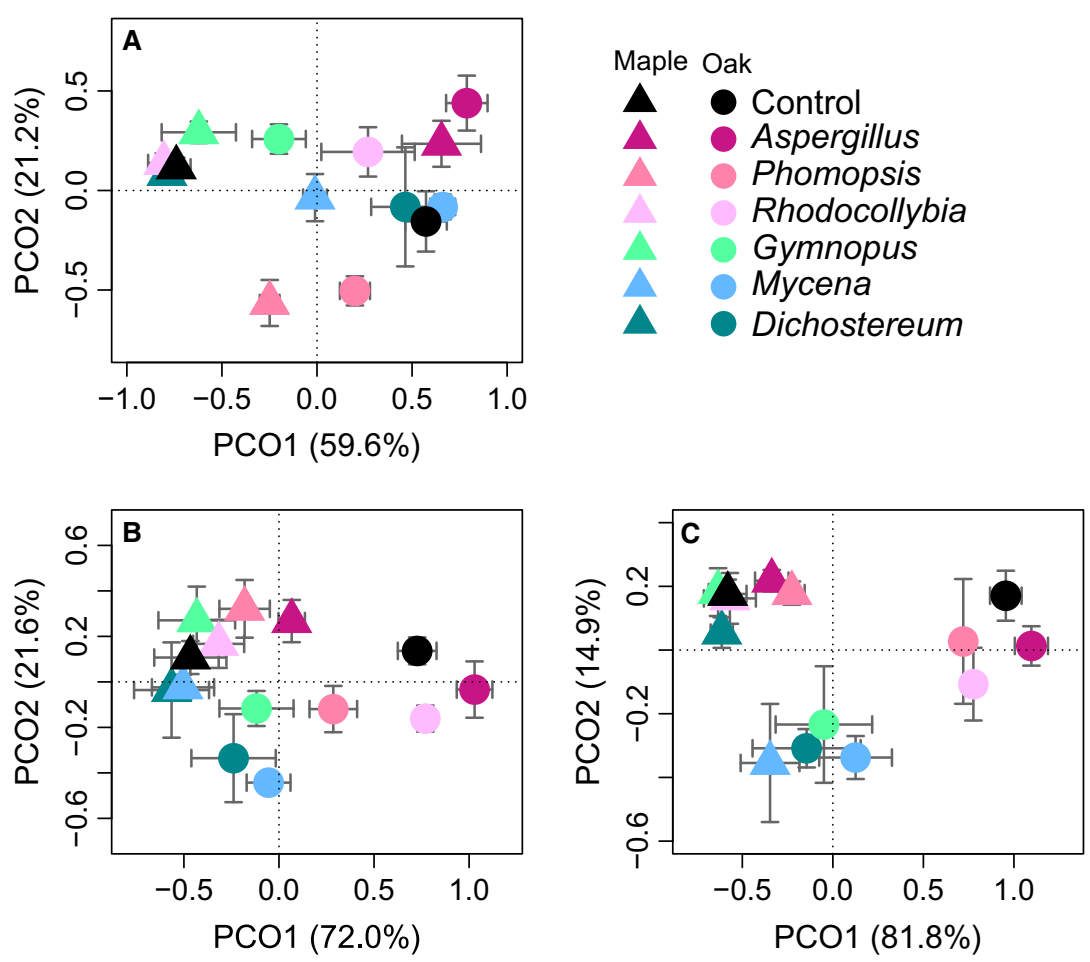

Fig. 4 Principal components analysis of potential enzyme activity after 1 month (A), 3 months (B) and 8 months (C). The Euclidean distance metric was used to calculate pairwise treatment differences in log-transformed enzyme potential at each time point. Error bars denote standard error between replicates within a treatment. Across all time points, PCo1 negatively correlated with $\beta$-glucosidase, $\mathrm{N}$-acetylaminoglucosidase and cellobiohydrolase potential activity $(r=-0.69$ to $-0.93, P<0.0001)$. $\mathrm{PCo} 2$ in Fig. $4 \mathrm{C}$ is negatively correlated with lignolytic activity $(r=-0.99 ; P<0.0001)$. 
porting information, Fig. 3). These observations collectively indicated that initial fungal colonizers had a significantly larger effect on community assembly of the energy-poor oak leaves.

\section{Role of initial colonizer history on community assembly through time}

To test the hypothesis that the importance of initial colonization attenuated through time, we compared effects sizes $\left(\eta_{p}^{2}\right)$ for compositional and functional characteristics of fungal communities at 1,3 and 8 months (Table 1). The initial colonist accounted for substantial and relatively consistent variation in fungal $\beta$-diversity; whereas, the impact of the initial colonizer on enzyme potential declined, suggesting that the initial colonist was less important in determining trajectories of metabolic potential through time. Interestingly, the initial fungal colonist appeared increasingly important in determining rates of decay, as identity of the initial colonist accounted for increasing variance of mass loss at later time points (Table 1). Despite the substantial role of initial colonizer in shaping community composition, results indicated that subsequent functional consequences are dependent on stage of community assembly.

\section{Initial colonizer traits and consequences to community assembly}

Mantel correlations tested the hypothesis that initial colonization of closely related fungal taxa would result in the assembly of similar communities over time. Following one month of community assembly, $\beta$-diversity of maple litter communities was significantly related to phylogenetic distances between fungal colonists $\left(R_{\text {Mantel }}\right.$ $=0.62, P=0.032)$, but not in oak litter communities $\left(R_{\text {Mantel }}=0.40, P=0.14\right)$. After 3 months, phylogenetic distance between initial colonists was marginally correlated with $\beta$-diversity in oak litter communities $\left(R_{\text {Mantel }}=0.33, P=0.08\right)$, but not maple litter communities $\left(R_{\text {Mantel }}=0.23, P=0.24\right)$. After 8 months, variation in colonist phylogenetic distance was not related to the $\beta$-diversity of oak litter communities $\left(R_{\text {Mantel }}=-0.09\right.$, $P=0.54)$ or maple litter communities $\left(R_{\text {Mantel }}=0.32\right.$, $P=0.20)$. Although phylogenetic relatedness between colonists was not a perfect predictor of community assembly trajectories, phylogenetically similar colonists generally resulted in more similar communities when compared to distantly related colonists at early stages of decay on lignin-rich leaf litter.

To investigate whether particular physiological traits of initial colonists shaped community assembly, colonizer respiration, total enzyme activity and lig- nolytic activity were regressed against fungal community compositional and functional departures from the control. Across both litter types, initial colonists with higher rates of respiration (log-transformed) correlated with larger $\beta$-diversity following one month (Fig. 5, $\left.r^{2}=0.54, P=0.007\right)$, although no relationship occurred at later time points $(P=0.17-0.47)$. Further, total enzyme potential or lignolytic potential of initial colonists was not related to community dissimilarity at 1,3 or 8 months $(P=0.16-0.94)$. Total enzyme potential of initial colonizer was weakly correlated with mass remaining (normalized to the control) after 1 month $\left(r^{2}=0.28\right.$, $\left.F_{1,10}=3.93, \quad P=0.076\right) \quad$ and 3 months $\quad\left(r^{2}=0.27\right.$, $\left.F_{1,10}=3.65, \quad P=0.085\right)$, but not 8 months $(P=0.16)$. Together, results suggest that colonist respiration and total enzyme potential were important factors structuring early trajectories of community assembly.

\section{Discussion}

Initial colonization had important consequences for fungal community assembly, wherein the physiological traits of the initial colonist accounted for the early trajectories of community composition and rates of litter decay. Support for this comes from evidence that the initial colonist suppressed fungal community richness and enhanced litter decay. Similarly, the initial colonist generated different trajectories of community composition and metabolic potential relative to control commu-

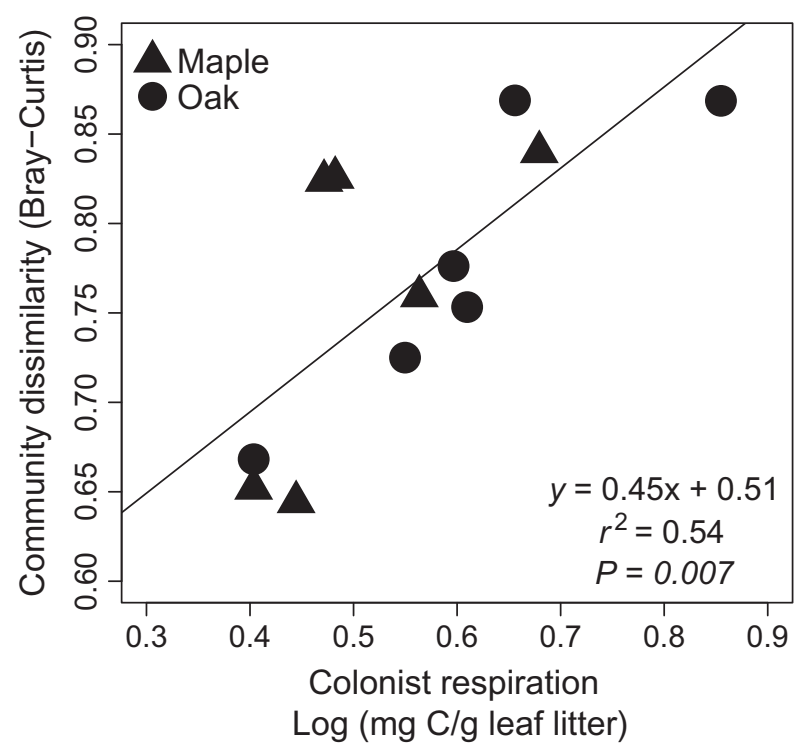

Fig. 5 Average Bray-Curtis dissimilarity of each initial colonizer history after one month, normalized to control, as a function of (log-transformed) respiration rate of initial colonizer. Simple linear regression revealed a significant relationship at $\alpha<0.05$. 
nities lacking an initial colonist, and most importantly, the degree of dissimilarity was highly dependent on the colonist's identity. Furthermore, during the early stages of community assembly ( 1 and 3 months), deviations from control community assembly were positively related to colonist respiration rate and metabolic potential to degrade plant detritus. Importantly, lignin-rich oak leaf litter generated increasingly divergent trajectories of community assembly, as initial colonizer identity resulted in increased $\beta$-diversity and a broader range of enzyme potentials in oak litter communities relative to maple litter communities. Together, our results indicate the important roles that physiological traits of initial colonists, as well as resource availability, play in shaping the balance between habitat filtering and initial colonization effects during the process of community assembly.

\section{Initial colonization altered community assembly}

Initial colonizer identity altered compositional and functional trajectories of fungal community assembly, indicating that initial colonization has important implications for biogeochemical cycling in soils. Providing support for this assertion, models of fungal $\beta$-diversity indicated that initial colonizer history accounted for differences in fungal community composition throughout the experiment (Table 1). Further, direct evidence for priority effects arose from increased initial colonizer abundance, relative to control communities (1 month, Table S5, Supporting information). Gaining early access to resources plausibly enhanced establishment success of fungal colonists, as the absence of competition did not require the production of energetically expensive secondary metabolites necessary for combative interactions (Holmer \& Stenlid 1997; Boddy 2000; Dickie et al. 2012), leading to niche pre-emption. However, no initial colonist ranked among the top OTUs driving differences between initial colonization treatments and control communities after 3 and 8 months of community assembly. Therefore, lasting consequences of initial colonization were not the result of high initial colonist abundance. Instead, initial colonizer identity may shape trajectories of community assembly by substrate modification and the subsequent suppression or enhancement of later propagule establishment (Fukami et al. 2010; Dickie et al. 2012; Ottosson et al. 2014).

While important insights can be gleaned from the importance of initial colonization events on fungal community assembly, an important limitation to our study is that we did not explicitly test priority effects. A true test of priority effects requires the ordered application of organisms, as well as direct quantification of individual competitive outcomes. While initial colonization altered trajectories of fungal community assembly, gaining a firm understanding the underlying mechanism will require further experimental manipulation.

Our results confirmed the hypothesis that characteristics of the initial colonizer significantly altered rates of litter decay and community enzymatic potential (Table 1), thereby providing evidence that initial colonization by saprotrophic fungi has important functional implications (Fukami et al. 2010; Dickie et al. 2012). First, initial colonizer identity altered functional characteristics in a manner that was generally consistent with changes in community composition, indicating that fungal communities are not functionally redundant (McGuire et al. 2010; Kivlin \& Treseder 2014). Second, decomposition by the assembling litter community appeared sensitive to initial colonization, as functional differences from control communities were dependent on the initial colonizer (Cleland et al. 2015). For example, certain initial colonists (i.e. Gymnopus, Мycena and Dichostereum) led to enhanced respiration and decomposition (Table S6, Supporting information, Fig. 3), with largest differences in enzyme activity apparent in communities initially colonized by lignolytic fungi (Fig. 4). While some observations suggest that functional characteristics may converge despite strong priority effects (e.g. Fukami et al. 2005; Petermann et al. 2010; Tan et al. 2012), our results indicate that the competitive advantage gained by certain initial fungal decomposers had important consequences for soil biogeochemical cycling and further necessitates investigation of the factors that strengthen priority effects.

\section{Impact of initial colonizer decreased over time}

While the initial fungal colonizer shaped community assembly and litter decay throughout the experiment, habitat filtering may become increasingly important at later stages of assembly (Ferrenberg et al. 2013). Despite accounting for a relatively stable amount of variance in fungal $\beta$-diversity and mass loss, the identity of initial colonizer explained less variation in enzyme potential through time. Concomitantly, litter type captured an increasing variation in enzyme potential through time, potentially indicating the growing importance of successional trajectories in shaping functional characteristics of communities. For example, the depletion of labile organic substrates may increase selection for organisms with the physiological capacity to decompose the lignified components of plant detritus (Hudson 1968; Frankland 1998; Di Lonardo et al. 2013). Second, the mechanism by which initial colonists shaped community assembly may change with time, as initial colonist respiration significantly accounted for deviations from community assembly - relative to controls - at early time points (Fig. 5). In early stages of community 
assembly, initial colonists may directly influence community traits simply due to their high abundance, whereas in later stages initial colonizers may alter establishment of later-arriving colonizers via prior resource consumption and subsequent niche modification ('impact niche'; Vannette \& Fukami 2014). The persistent influence of initial colonizer history indicates that initial colonizers alter the competitive dynamics of later establishing taxa, even after their direct influence dissipates (Ottosson et al. 2014). Furthermore, our results indicate that initial colonization effects are not mutually exclusive of habitat filtering, but rather mechanisms that interact to shape fungal community composition and function.

\section{As a habitat filter, litter type altered the influence of the initial fungal colonist}

Contrary to our hypothesis, consequences of initial fungal colonizers were stronger on oak leaves, a relatively lignin-rich and energy-poor substrate, when compared to maples leaves. Supporting this idea, dispersion analysis revealed that oak litter community composition was more variable, indicating initial colonizers elicited larger departures from control communities (Fig. 2B,C). Stronger consequences of initial colonizers were expected on maple leaves, due to evidence that high resource conditions enhanced the establishment success of plant initial colonists, leading to strong priority effects and increasingly divergent trajectories of community assembly (Ejrnæs et al. 2006; Kardol et al. 2013). Along this same line of thinking, drought reduced the importance of priority effects relative to habitat filtering as plant taxa were removed according to their tolerance to harsh conditions (Chase 2007; Leopold et al. 2015). Because our experimental 'low resource' environment generated wider ranges of community assembly, we believe it is important to recognize that resource availability is highly dependent on the physiological attributes of the organisms under consideration. Oak leaves, with high lignin content and longer residence times on the forest floor relative to maple leaves (Table S1, Supporting information; Melillo et al. 1982), could be considered a limited resource substrate to a sugar fungus. However, oak litter may represent an abundant resource for fungi capable of degrading more recalcitrant components of the plant cell wall, including lignin (Osono \& Takeda 2001; Voř́šsková \& Baldrian 2013). The larger variation in oak litter community assembly following initial colonization, combined with the largest differences elicited by isolates with high lignolytic capacity (e.g. Dichostereum, Gymnopus, Mycena; Fig. 2), suggests that relatively lignin-rich oak leaves may enhance the establishment success of relatively rare or slow-growing taxa, thereby increasing community divergence and subsequent decomposition (Pagaling et al. 2014). Due to additional differences in litter biochemistry between oak and maple leaves aside from lignin content (Preston \& Trofymow 2000), determining whether lignin is the biochemical attribute driving different initial colonization effects will require testing a range of substrates with varied lignin content. Nevertheless, our observations indicate that the interactions between habitat filtering and initial colonization determine outcomes of fungal community assembly.

\section{Community assembly was related to physiological traits of initial colonist}

Colonist respiration rate, enzyme potential and evolutionary history were important determinants of fungal community composition and functional characteristics. Because initial colonization by close phylogenetic relatives resulted in similar competitive outcomes when considering fungal community composition on oak leaves, the phylogenetic context may be useful to understanding consequences of priority effects on microbial community composition and function under certain environmental conditions (Peay et al. 2012; Tan et al. 2012). Second, community assembly during the earliest stage of decomposition appeared dependent on respiration rate of the initial colonizer (Fig. 5), indicating that rapidly respiring colonists, such as Phomopsis, gained a competitive advantage in early stages of community assembly. Third, the total enzyme potential of initial colonizers weakly correlated with mass of litter lost (normalized to control) after one and three months. This result supports the idea that the metabolic potential of initial colonists altered decomposition rates, due to changes in the composition of assembling communities that resulted from successful colonist establishment. Relative to control treatments, the largest departures in community composition and litter decay of litter communities arose following initial colonization by Mycena, Gymnopus and Dichostereum, all basidiomycetes capable of decomposing lignin. While no linear relationship occurred between colonist lignolytic potential and community departure from control communities, our observations indicate that ability to metabolize lignin may be one of several factors that determines successful fungal establishment and the strength of initial colonization effects in saprotrophic communities.

\section{Conclusion}

The importance of the stochastic sequence and timing of propagules may hinder our ability to predict 
outcomes of community assembly (Dickie et al. 2012). Here, we have demonstrated that an initial colonizer can alter the community composition and functional characteristics of assembling saprotrophic fungi. However, we also present evidence that the strength of these initial colonization effects on fungal community composition and decomposition rate change through time and are dependent upon substrate availability and physiological traits within a regional species pool. As a result, identifiable ecological mechanisms appear to underlie the seemingly stochastic consequences of priority effects (Vannette \& Fukami 2014). Investigation of the factors that alter dispersal and establishment success of organisms is necessary for a comprehensive understanding of factors that influence strength of priority effects (Johnson 2015), and ultimately, the factors that structure community assembly. Furthermore, as regulators of biogeochemical cycling in soils, our results suggest that understanding the mechanisms by which priority effects structure fungal community assembly may be key to understanding drivers of ecosystem-level processes.

\section{Acknowledgments}

The Department of Energy's Biological and Environmental Research Division, as well as the National Science Foundation's Graduate Research Fellowship Program and Long-Term Research in Environmental Biology Program supported this research. The authors would like to thank Will Argiroff, Jules Cooch, Remy Long, Karl Romanowicz and Sydney Salley for their invaluable laboratory and field assistance. Rima Upchurch was essential in her logistical and intellectual support.

\section{References}

Allison SD (2012) A trait-based approach for modelling microbial litter decomposition. Ecology Letters, 15, 1058-1070.

Belyea LR, Lancaster J (1999) Assembly rules within a contingent ecology. Oikos, 86, 402-416.

Boddy L (2000) Interspecific combative interactions between wood-decaying basidiomycetes. FEMS Microbiology Ecology, 31, 185-194.

Chase JM (2003) Community assembly: when should history matter? Oecologia, 136, 489-498.

Chase JM (2007) Drought mediates the importance of stochastic community assembly. Proceedings of the National Academy of Sciences of the United States of America, 104, 17430-17434.

Claesson MJ, O'Sullivan O, Wang Q et al. (2009) Comparative analysis of pyrosequencing and a phylogenetic microarray for exploring microbial community structures in the human distal intestine. PLOS ONE, 4, e6669.

Cleland EE, Esch E, McKinney J (2015) Priority effects vary with species identity and origin in an experiment varying the timing of seed arrival. Oikos, 124, 33-40.

Cline LC, Zak DR (2014) Dispersal limitation structures fungal community assembly in a long-term glacial chronosequence. Environmental Microbiology, 16, 1538-1548.
Cole JR, Wang Q, Fish JA et al. (2014) Ribosomal Database Project: Data and tools for high throughput rRNA analysis. $\mathrm{Nu}$ cleic Acids Research, 42, 633-642.

Di Lonardo DP, Pinzari F, Lunghini D et al. (2013) Metabolic profiling reveals a functional succession of active fungi during the decay of Mediterranean plant litter. Soil Biology $\mathcal{E}$ Biochemistry, 60, 210-219.

Diamond JM (1975) Assembly of Species Communities. In: Ecology and Evolution of Communities(eds Cody ML, Diamond JM), pp. 342-444. Harvard University Press, Cambridge, MA.

Dickie IA, Fukami T, Wilkie JP, Allen RB, Buchanan PK (2012) Do assembly history effects attenuate from species to ecosystem properties? A field test with wood-inhabiting fungi. Ecology Letters, 15, 133-141.

Drake JA (1991) Community-assembly mechanics and the structure of an experimental species ensemble. The American Naturalist, 137, 1-26.

Edgar RC, Haas BJ, Clemente JC, Quince C, Knight R (2011) UCHIME improves sensitivity and speed of chimera detection. Bioinformatics, 27, 2194-2200.

Edwards IP, Zak DR (2011) Fungal community composition and function after long-term exposure of northern forests to elevated atmospheric $\mathrm{CO} 2$ and tropospheric O3. Global Change Biology, 17, 2184-2195.

Ejrnæs R, Bruun HH, Graae BJ (2006) Community assembly in experimental grasslands: Suitable environment or timely arrival? Ecology, 87, 1225-1233.

Entwistle EM, Zak DR, Edwards IP (2013) Long-term experimental nitrogen deposition alters the composition of the active fungal community in the forest floor. Soil Science Society of America Journal, 77, 1648-1658.

Ferrenberg S, O'Neill SP, Knelman JE et al. (2013) Changes in assembly processes in soil bacterial communities following a wildfire disturbance. The ISME Journal, 7, 1102-1111.

Floudas D, Binder M, Riley R et al. (2012) The Paleozoic origin of enzymatic lignin decomposition reconstructed from 31 fungal genomes. Science, 336, 1715-1719.

Frankland JC (1998) Fungal succession - unravelling the unpredictable. Mycological Research, 102, 1-15.

Fukami T, Bezemer TM, Mortimer SR, van der Putten WH (2005) Species divergence and trait convergence in experimental plant community assembly. Ecology Letters, 8, 1283-1290.

Fukami T, Dickie IA, Paula Wilkie J et al. (2010) Assembly history dictates ecosystem functioning: evidence from wood decomposer communities. Ecology Letters, 13, 675-684.

Goering HK, Van Soest PJ (1970) Forage Fiber Analysis. USDA Agricultural Research Service Handbook Number 379. U.S. Dept. of Agriculture, Washington, DC.

Good IJ (1953) The population frequencies of species and the estimation of population parameters. Biometrika, 40, 237-264.

Hatakka A (1994) Lignin-modifying enzymes from selected white-rot fungi: production and role in lignin degradation. FEMS Microbiology Reviews, 13, 125-135.

He X, Lin Y, Han G, Guo P, Tian X (2010) The effect of temperature on decomposition of leaf litter from two tropical forests by a microcosm experiment. European Journal of Soil Biology, 46, 200-207.

Hiscox J, Savoury M, Müller CT et al. (2015) Priority effects during fungal community establishment in beech wood. The ISME Journal, (in press). doi:10.1038/ismej.2015.38. 
Holmer L, Stenlid J (1997) Competitive hierarchies of wood decomposing basidiomycetes in artificial systems based on variable inoculum sizes. Oikos, 79, 77-84.

Hudson HJ (1968) The ecology of fungi on plant remains above the soil. New Phytologist, 67, 837-874.

Johnson D (2015) Priorities for research on priority effects. New Phytologist, 205, 1375-1377.

Kahm M, Hasenbrink G, Lichtenberg-Frate H et al. (2010) grofit: Fitting Biological Growth Curves with R. Journal of Statistical Software, 33, 1-21. Avaialble from: http://www.jstatsoft. org/v33/i07/.

Kardol P, Souza L, Classen AT (2013) Resource availability mediates the importance of priority effects in plant community assembly and ecosystem function. Oikos, 122, 84-94.

Kennedy PG, Bruns TD (2005) Priority effects determine the outcome of ectomycorrhizal competition between two Rhizopogon species colonizing Pinus muricata seedlings. New Phytologist, 166, 631-638.

Kivlin SN, Treseder KK (2014) Initial phylogenetic relatedness of saprotrophic fungal communities affects subsequent litter decomposition rates. Microbial Ecology, 69, 748-757.

Körner C, Stöcklin J, Reuther-Thiébaud L, Pelaez-Riedl S (2008) Small differences in arrival time influence composition and productivity of plant communities. New Phytologist, 177, 698-705.

Lakens D (2013) Calculating and reporting effect sizes to facilitate cumulative science: A practical primer for $\mathrm{t}$-tests and ANOVAs. Frontiers in Psychology, 4, 1-12.

Langenheder S, Prosser JI (2008) Resource availability influences the diversity of a functional group of heterotrophic soil bacteria. Environmental Microbiology, 10, 2245-2256.

Langenheder S, Székely AJ (2011) Species sorting and neutral processes are both important during the initial assembly of bacterial communities. The ISME Journal, 5, 10861094.

Leopold DR, Tanentzap AJ, Lee WG, Heenan PB, Fukami T (2015) Evolutionary priority effects in New Zealand alpine plants across environmental gradients. Journal of Biogeography, 42, 729-737.

Lewontin RC (1969) The Meaning of stability. Brookhaven Symposia in Biology, 22, pp. 13-24. Brookhaven, NY.

Liu K-L, Porras-Alfaro A, Kuske CR, Eichorst SA, Xie G (2012) Accurate, rapid taxonomic classification of fungal large-subunit rRNA genes. Applied and environmental microbiology, 78, 1523-1533.

Martiny JBH, Eisen JA, Penn K, Allison SD, Horner-Devine CM (2011) Drivers of bacterial $\beta$-diversity depend on spatial scale. Proceedings of the National Academy of Sciences of the United States of America, 108, 7850-7854.

McGuire KL, Bent E, Borneman J et al. (2010) Functional diversity in resource use by fungi. Ecology, 91, 2324-2332.

McMurdie PJ, Holmes S (2014) Waste not, want not: why rarefying microbiome data is inadmissible. PLoS Computational Biology, 10, e1003531.

Melillo JM, Aber JD, Muratore JF (1982) Nitrogen and lignin control of hardwood leaf litter decomposition dynamics. Ecology, 63, 621-626.

Nakagawa S, Cuthill IC (2007) Effect size, confidence interval and statistical significance: A practical guide for biologists. Biological Reviews, 82, 591-605.
Nemergut DR, Schmidt SK, Fukami T et al. (2013) Patterns and processes of microbial community assembly. Microbiology and Molecular Biology Reviews, 77, 342-356.

Oksanen JF, Blanchet G, Kindt R et al. (2015) vegan: Community Ecology Package. $\mathrm{R}$ package version 2.3-0. Available form: http:/ /CRAN.R-project.org/package=vegan.

Osono T, Takeda H (2001) Organic chemical and nutrient dynamics in decomposing beech leaf litter in relation to fungal ingrowth and succession during 3-year decomposition processes in a cool temperate deciduous forest in Japan. Ecological Research, 16, 649-670.

Ottosson E, Nordén J, Dahlberg A et al. (2014) Species associations during the succession of wood-inhabiting fungal communities. Fungal Ecology, 11, 17-28.

Pagaling E, Strathdee F, Spears BM et al. (2014) Community history affects the predictability of microbial ecosystem development. The ISME Journal, 8, 19-30.

Peay KG, Belisle M, Fukami T (2012) Phylogenetic relatedness predicts priority effects in nectar yeast communities. Proceedings of the Royal Society B: Biological Sciences, 279, 749-758.

Petermann JS, Fergus AJF, Roscher C et al. (2010) Biology, chance, or history? The predictable reassembly of temperate grassland communities. Ecology, 91, 408-421.

Porter TM, Golding GB (2012) Factors that affect large subunit ribosomal DNA amplicon sequencing studies of fungal communities: classification method, primer choice, and error. PLoS ONE, 7, e35749.

Preston CM, Trofymow JA, Working Group the CIDE (2000) Variability in litter quality and its relationship to litter decay in Canadian forests. Canadian Journal of Botany, 78, 12691287.

Saiya-Cork KR, Sinsabaugh RL, Zak DR (2002) The effects of long term nitrogen deposition on extracellular enzyme activity in an Acer saccharum forest soil. Soil Biology and Biochemistry, 34, 1309-1315.

Schloss PD, Gevers D, Westcott SL (2011) Reducing the effects of PCR amplification and sequencing artifacts on 16S rRNAbased studies. PLoS ONE, 6, e27310.

Tan J, Pu Z, Ryberg WA, Jiang L (2012) Species phylogenetic relatedness, priority effects, and ecosystem functioning. Ecology, 93, 1164-1172.

Travers KJ, Chin CS, Rank DR, Eid JS, Turner SW (2010) A flexible and efficient template format for circular consensus sequencing and SNP detection. Nucleic Acids Research, gkq543.

Tucker CM, Fukami T (2014) Environmental variability counteracts priority effects to facilitate species coexistence: evidence from nectar microbes. Proceedings of the Royal Society B: Biological Sciences, 281, 20132637.

Van der Wal A, Geydan TD, Kuyper TW, de Boer W (2013) A thready affair: linking fungal diversity and community dynamics to terrestrial decomposition processes. FEMS Microbiology Reviews, 37, 477-494.

Vannette RL, Fukami T (2014) Historical contingency in species interactions: towards niche-based predictions. Ecology Letters, 17, 115-124.

Vilgalys R, Hester M (1990) Rapid genetic identification and mapping of enzymatically amplified ribosomal DNA from several Cryptoccocus species. Journal of Bacteriology, 172, 4238-4246. 
Voříšková J, Baldrian P (2013) Fungal community on decomposing leaf litter undergoes rapid successional changes. The ISME Journal, 7, 477-486.

Wilbur HM, Alford RA (1985) Priority Effects in Experimental Pond Communities : Responses of Hyla to Bufo and Rana. Ecology, 66, 1106-1114.

Zwietering $\mathrm{MH}$, Jongenburger I, Rombouts FM, van't Riet $\mathrm{K}$ (1990) Modeling of the bacterial growth curve. Applied and Environmental Microbiology, 56, 1875-1881.

L.C.C. and D.R.Z. designed the experiment and analysed data. L.C.C. performed analyses and wrote first draft of study. D.R.Z. contributed substantially to manuscript revisions.

\section{Data accessibility}

DNA sequences can be accessed at the NCBI Sequence Read Archive under study Accession no. SRP056628. Alignment files, tree files and OTU tables can be accessed at Dryad under doi:10.5061/dryad.r3b5d, in addition to community enzyme, respiration and mass data.

\section{Supporting information}

Additional supporting information may be found in the online version of this article.

Table S1 Litter biochemistry of Acer saccharum (sugar maple) and Quercus rubra (red oak) leaves collected for use in microcosm experiment.

Table S2 Accession numbers and taxonomy of references sequences used for sequence alignment and phylogenetic tree construction.

Table S3 Effect size $\left(\eta_{\mathrm{p}}{ }^{2}\right)$ from PerMANOVA models at each experimental time point using un-rarefied data.

Table S4 Alpha and beta diversity calculations for each treatment combination, averaged across biological replicates within each treatment.

Table S5 Similarity Percentage analysis (SIMPER), calculated the contribution of OTUs towards the community dissimilarity between each initial colonizer history and the control, for each time point (1,3 and 8 months) and litter type (oak and maple).

Table S6 Using the sigmoidal Gompertz model y $(\mathrm{t})=\mathrm{A} \quad \times$ $\exp [-\exp \mathrm{u}-\mathrm{e} / \mathrm{A}(\lambda-\mathrm{t})+1)]), \lambda, \mu$ and $A$ were calculated from the cumulative respiration in each treatment combination $(n=10)$ through time. 